\title{
Gene expression signature in adipose tissue of acromegaly patients
}

\author{
Irit Hochberg ${ }^{1,3^{*}}$, Quynh T Tran², Ariel R Barkan ${ }^{3}$, Alan R Saltiel ${ }^{4}$, William F Chandler ${ }^{5}$, Dave Bridges ${ }^{3,6,7}$ \\ From UT-KBRIN Bioinformatics Summit 2014 \\ Cadiz, KY, USA. 11-13 April 2014
}

\section{Background}

Acromegaly is a rare endocrine disorder with excess growth hormone $(\mathrm{GH})$ production. This disorder has important metabolic effects in insulin resistance and lipolysis. The objective of this study was to explore transcriptional changes induced by GH in adipose tissue.

\section{Materials and methods}

Patients with acromegaly $(\mathrm{n}=9)$ or non-functioning pituitary adenoma $(n=11)$ were prospectively observed from March 2011 to June 2012. The patients underwent clinical and metabolic profiling including assessment of HOMA-IR. Explants of adipose tissue were assayed exvivo for lipolysis and ceramide levels. Adipose tissue was analyzed by RNA sequencing (RNAseq).

\section{Results}

There was evidence of reduced insulin sensitivity based on the increase in fasting glucose, insulin and HOMA-IR score. We observed several previously reported transcriptional changes (IGF1, IGFBP3) as well as several novel transcriptional changes, some of which may be important for GH signal regulation (PTPN3 and PTPN4) and the effect of GH on growth and proliferation. Several transcripts could potentially be important in $\mathrm{GH}$-induced metabolic changes. Specifically, induction of $L P L$, $A B H D 5, A C V R 1 C$ could contribute to enhanced lipolysis and may explain the suggestive enhancement of adipose tissue lipolysis in acromegaly patients as reflected by glycerol release from the explants of the two groups of patients $(\mathrm{p}=0.09)$. Higher expression of $S C D$ and TCF7L2 could contribute to insulin resistance. Expression of
$H S D 11 B 1$ was reduced and $G R$ was increased, predicting modified glucocorticoid activity in acromegaly.

\section{Conclusions}

We identified the acromegaly gene expression signature in human adipose tissue. The significance of altered expression of specific transcripts will enhance our understanding of the metabolic and proliferative changes associated with acromegaly.

\section{Authors' details}

${ }^{1}$ Institute of Endocrinology, Diabetes and Metabolism, Rambam Health Care Campus, Haifa, Israel. ${ }^{2}$ Department of Preventive Medicine, University of Tennessee Health Science Center, Memphis, TN 38163, USA. ${ }^{3}$ Department of Internal Medicine, University of Michigan, Ann Arbor, Ml 48109, USA. ${ }^{4}$ Life Sciences Institute, University of Michigan, Ann Arbor, MI 48109, USA. ${ }^{5}$ Neurosurgery, University of Michigan, Ann Arbor, MI 48109, USA.

${ }^{6}$ Department of Physiology, University of Tennessee Health Science Center, Memphis, TN 38163, USA. 'Children's Foundation Research Institute, Le Bonheur Children's Hospital, Memphis, TN 38103, USA.

Published: 29 September 2014

doi:10.1186/1471-2105-15-S10-P28

Cite this article as: Hochberg et al:: Gene expression signature in adipose tissue of acromegaly patients. BMC Bioinformatics 2014 15(Suppl 10):P28.

\footnotetext{
* Correspondence: i_hochberg@rambam.health.gov.il

'Institute of Endocrinology, Diabetes and Metabolism, Rambam Health Care

Campus, Haifa, Israel

Full list of author information is available at the end of the article
} 